e-Neuroforum $2010 \cdot 1: 81-88$

DOI 10.1007/s13295-010-0010-1

(C) Springer-Verlag 2010

\author{
J. Schmidt \\ Department of Neurology, Göttingen
}

\title{
Of amyloid and inflammation: causes of chronic muscle disease
}

patients in particular. It is clearly distinct from other inflammatory muscle diseases such as dermatomyositis and polymyositis. Distinguishing characteristics include predominant involvement of shoulder girdle and pelvis muscles, which is not a main feature of sIBM. Dermatomyositis and polymyositis often present at younger ages, sometimes even in adolescence, and usually respond well to immunosuppression or immunomodulatory therapy. The main difference, however, lies in the clearly differing pathology as seen on biopsy. Only in sIBM can a typical formation of vacuoles (so-called red rimmed vacuoles) and inclusion bodies be seen in addition to significant inflammation (• Fig. 1).

Alongside the most common forms of sIBM there are hereditary forms of the disease. A mutation on the UDP-Nacetylglucosamine 2-epimerase/ $\mathrm{N}$-acetylmannosamine kinase gene is responsible for autosomal-recessive IBM. An autosomal dominant hereditary form is caused by a mutation in valosin containing protein and is associated with memory loss and bone disease. These generally very rare hereditary forms of IBM, howly affected. As a result, these patients experience progressive loss of strength in the legs with increasingly restricted ability to stand and walk. In addition, patients gradually lose the strength to form a fist and make targeted, fine finger movements. The oesophagus is often also involved, causing swallowing and eating difficulties. After a 5-year course, patients often depend on mobility aides, sometimes even a wheelchair. Quality of life is in general significantly reduced by the disease and no effective therapy is available to date. Various studies report that no significant improvement in muscle strength could be seen when medication to reduce or modulate the inflammatory reaction in muscle, such as immunoglobulins, glucocorticosteroids, azathioprine and methotrexate, was given [9].

The first losses in strength as a result of sIBM are often seen after the age of 50 . With a prevalence of over 50 per million, the disease belongs in the rare-disease category. However, sIBM is the most frequently acquired muscle disorder in older



Fig. 1 Trichrome histochemistry of a muscle biopsy in an sIBM patient. Muscle cells entirely surrounded by inflammatory cells can be seen (arrow). At the same time, muscle fibres with typical red rimmed vacuoles and inclusion bodies are visible (arrowhead in magnified insert) 
a

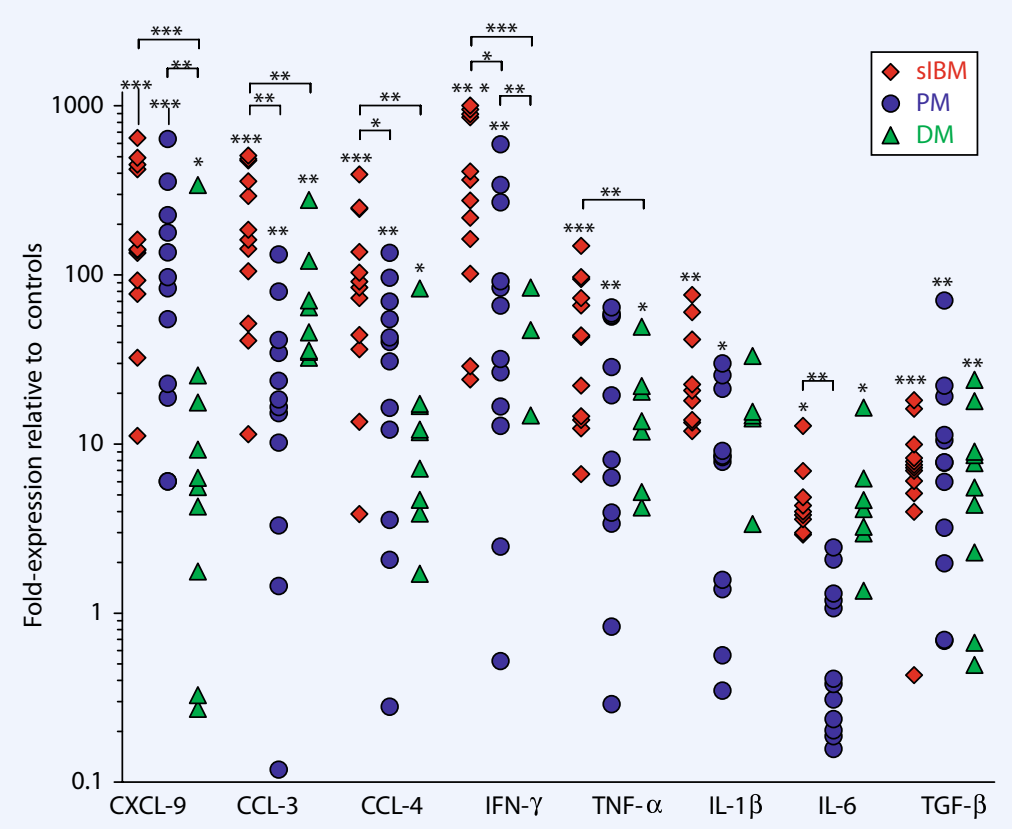

b


Fig. $2 \triangle$ a mRNA expression in relation to GAPDH by means of quantitative PCR in sIBM patients $(n=12)$, polymyositis $(P M ; n=12)$ and dermatomyositis $(D M ; n=11)$. Marker expression in relation to non-myopathic controls $(n=12) .{ }^{*} p<0.05,{ }^{* *} p<0.01$ and ${ }^{* * *} p<0.001$. b Immunohistochemical double and serial staining for MHC-I, CXCL-9, CCL-3 and IL- $1 \beta$. The staining signal in muscle in sIBM patients is found mainly in muscle fibres, the signal in dermatomyositis is found mainly in connective tissue cells and capillaries, while it remains lower in polymyositis and restricted to individual areas with increased inflammation. (Images obtained from the open-access publication [23] with permission)

ever, are clearly distinct from the sporadic variants, partly due to a completely different distribution pattern in affected muscles and partly due to the absence of inflammation in tissue samples.

\section{Inflammatory cells in} skeletal muscle

Certain surface molecules which have their own individual signature are present in greater numbers in sIBM, in particular so-called HLA (human leukocyte antigen)-B8 and HL-DR3. A similar accumulation of certain HLA molecules is seen predominantly in classic autoimmune diseases such as rheumatoid arthritis or multiple sclerosis. This is an indication that specific autoimmune mechanisms play a role in sIBM. Moreover, the onset of other autoimmune diseases of the thyroid or connective tissue, e.g. rheumatoid arthritis, is often concomitant to sIBM. Severe inflammation and production of various inflammatory mediators [cytokines such as tumor necrosis factor (TNF)- $\alpha$ and chemokines such as chemokine ligand (CXC-L)-9] can be seen in sIBM muscle. While these mediators can cause direct damage to muscle fibres on the one hand, particular cells in the immune system are called into the muscle, such as macrophages and T-leukocytes as well as B-lymphocytes, on the other. A large number of studies were able to show that there is an increase in T-cells in the skeletal muscle in the case of sIBM, as in polymyositis. This type of immune cell division in inflamed tissue is called "local clonal expansion", since the cells originate clone-like from a single original cell. Using the very elegant and highly sensitive method of spectratyping, with initial PCR followed by high-resolution electrophoresis, clonal expansion could also be observed in sIBM [7][21]. This kind of analysis is virtually able to record the "genetic fingerprint" of all T-cells found in the relevant sample. To evoke a sufficiently strong and specific growth signal in a T-cell, certain surface molecules need to bind to the lymphocytes. This sort of specific interaction is usually reserved for specialized, antigen-presenting cells such as dendritic cells: Following metabolization of damaged muscle fibres for example, these cells are able to deliver appropriate protein molecules on the surface in order to effect an activation of T-cells against precisely this molecule. In the case of sIBM, as in other autoimmune disease, a similar activation of T-cells can also take place in the 
inflamed tissue itself. In muscle, this antigen presentation can be taken over by the muscle fibres themselves. As shown by the work of Heinz Wiendl as well as our own data, muscle fibres themselves possess the relevant molecules to activate T-cells [22] [28]. In addition to the expression of major histocompatibility complex (MHC), which is present in almost every fibre in sIBM, in contrast to healthy muscle, costimulatory factors also play an important role. It could be shown that the co-stimulatory molecule inducible co-stimulator (ICOS) and its ligand (ICOS-L) are upregulated in muscle in the case of sIBM. Moreover, it was observed that ICOS-L on muscle fibres came into contact with celldamaging, cytotoxic (CD8-positive) Tcells. Corresponding "co-stimulation" via ICOS could also influence the destructiveness of cytotoxic T-cells.

Thus, muscle fibres in sIBM could be involved in all three steps of T-cell autoimmunity: (1) Recruitment via secretion of mediators; (2) local antigen presentation; (3) activation of T-lymphocytes [29].

\section{The development of $\beta$-amyloid in sIBM}

In addition to the marked inflammatory reaction seen in SIBM, there is an accumulation of defective proteins in the muscle. At the same time, all the molecules which play a role in Alzheimer's, Parkinson's and Huntington's disease, e.g. tau, ubiquitin, APO-E and $\alpha$-synuclein, occur in sIBM [1]. The most commonly occurring protein is $\beta$-amyloid, which is deposited in the brain primarily in Alzheimer's disease and is cleaved from the precursor molecule amyloid precursor protein (APP) by secretases. In contrast to Alzheimer's disease, where $\beta$-amyloid is mainly found extracellularly as plaques, it remains within the muscle fibres in SIBM. In a cell culture model, overexpression of APP lead to the development of vacuoles and the subsequent degeneration of muscle cells [2]. Also, in genetically altered mice, increased development of APP led to an accumulation of $\beta$-amyloid and pathological changes to the skeletal muscles, closely resembling IBM [12][24]. A dysfunction of the ubiquitin-proteasome system has already been considered as a principal cause of this accumulation of pathological molecules [1]. This is one of two significant cascades which establish an equilibrium between required proteins and proteins no longer needed, whereby older proteins are marked by ubiquitin and degraded via the proteasome pathway. In general, failure of this mechanism leads to an undesired accumulation of proteins, which cause other dysfunctions or cell damage within the cells. By developing compartments such as vacuoles or inclusion bodies, the damage potential of these proteins can be reduced.

A second mechanism for the degradation of a cell's own proteins is autophagy, a highly conserved cell process [15]. Autophagy is a lysosomal process whereby protein degradation takes place within vesicles formed by a double membrane which can enclose both free proteins and whole organelles. The vesicles then fuse with lysosomes which contain the necessary enzymes to degrade the proteins contained in the autophagic vesicle and make the remnants of the cell available for re-use, much like in a recycling process. Autophagy is indispensable for a cell in order to prevent unwanted proteins accumulating in the cell and possibly merging to form harmful aggregates. Autophagic mechanisms also play a particular role in neurodegenerative diseases where an accumulation of unwanted proteins is significant in the development of the disease [10][13][20]. New data from our group have shown that the vacuoles in sIBM muscle are also autophagic vesicles, which appear to play a role in the formation of $\beta$-amyloid [14]. A significant marker for autophagic activity is light-chain (LC) protein-3, since this is involved in the development of vacuoles and can be detected by means of immunoblotting. It could initially be shown that GFPLC-3 accumulated in muscle cells after the addition of chloroquine. This demonstrated that macroautophagy in muscle cells is continuously present. In the autophagic vacuoles, only the cell's own APP could be detected, none from external ovalbumin. In sIBM patients, 7.2\% LC-3-positive fibres were found compared with $1.4 \%$ in healthy muscle. Although other typical vacuolar muscle diseases, such as Pompe disease with $32.9 \%$, had a significantly higher level of vacuolar fibres, only in sIBM could a
e-Neuroforum 2010 - 1:81-88

DOI 10.1007/s13295-010-0010-1

C) Springer-Verlag 2010

\section{J. Schmidt \\ Of amyloid and inflammation: causes of chronic muscle disease}

Abstract

Classic inflammatory diseases of the skeletal muscle such as polymyositis and dermatomyositis often lead to rapid weakening of the arm and leg muscles. Inflammation in the muscle is caused primarily by immune cells and antibodies. Most patients respond well to standard immunosuppressive therapy. In sporadic inclusion body myositis (sIBM), similar pathomechanisms play a role, including cytotoxic T-lymphocytes, which attack and damage muscle fibers. At the same time, sIBM is characterized by an accumulation of aberrant molecules, particularly $\beta$-amyloid, which play a role in neurodegenerative diseases. This degeneration with formation of inclusion bodies and vacuoles may be the cause of the slow yet relentlessly progressive damage to the skeletal muscle and the lack of treatment efficacy of standard immunosuppression. Recent reports demonstrate that there is a specific interrelationship between inflammation with generation of mediators such as interleukin (IL)- $1 \beta$ and the amyloid-associated degeneration. The molecular mechanisms discussed here are important for the future design of better treatment strategies for chronic muscle inflammation. Furthermore, these mechanisms can contribute to a better understanding of the pathogenesis of neurodegenerative diseases.

\section{Keywords}

Inflammation, muscle · Cell stress · $\beta$-amyloid protein - Myopathies · Autophagy · T-cells 


\section{Review article}

a
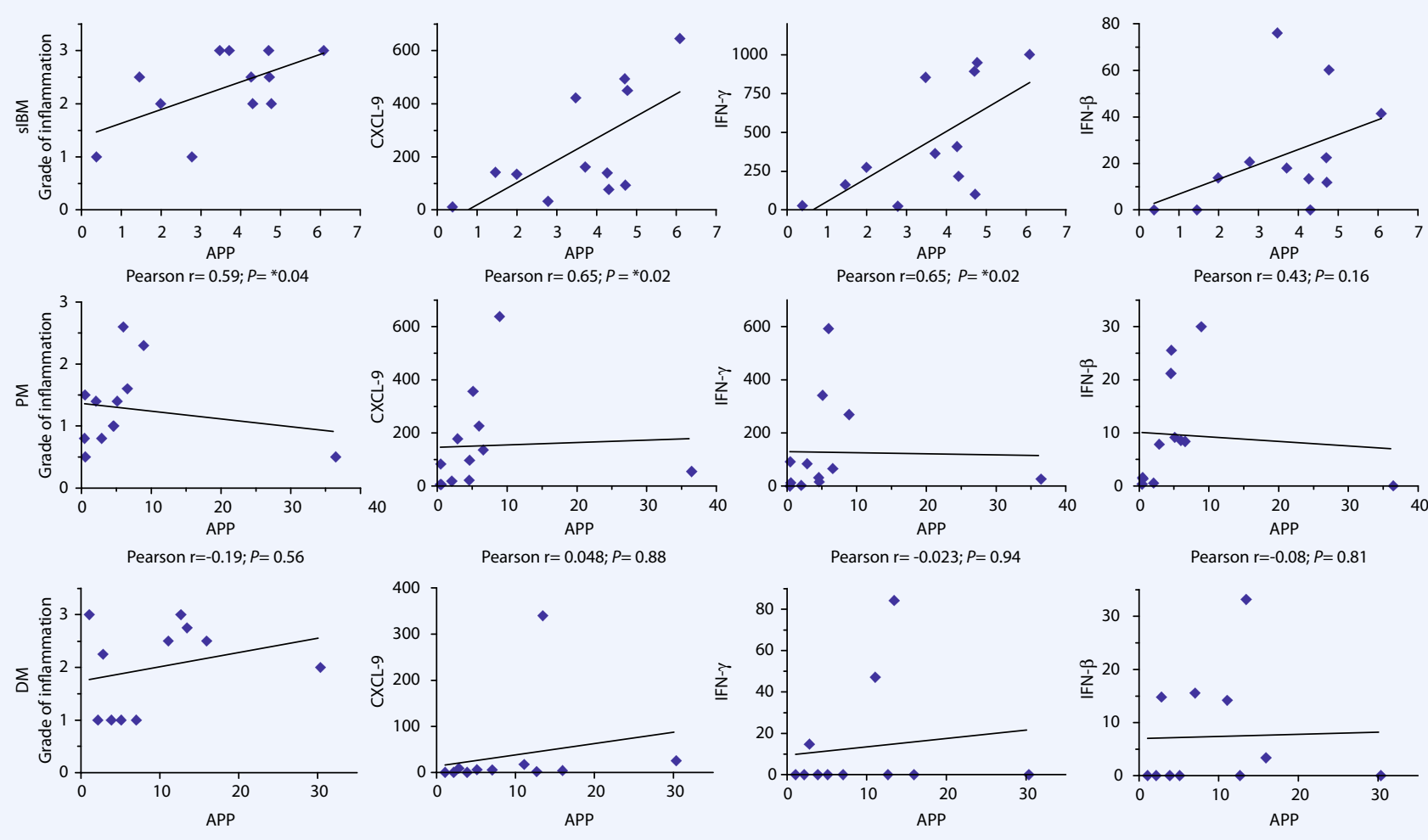

Pearson $\mathrm{r}=-0.023 ; P=0.94$

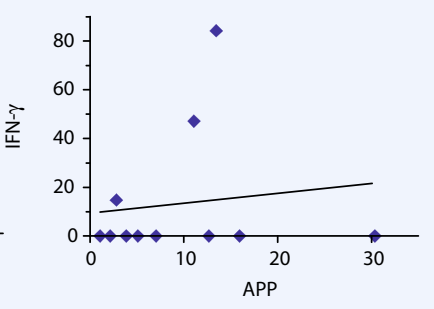

Pearson $r=-0.08 ; P=0.81$

Pearson $\mathrm{r}=0.28 ; P=0.41$

Pearson $\mathrm{r}=0.21 ; P=0.54$

Pearson $\mathrm{r}=0.13 ; P=0.71$

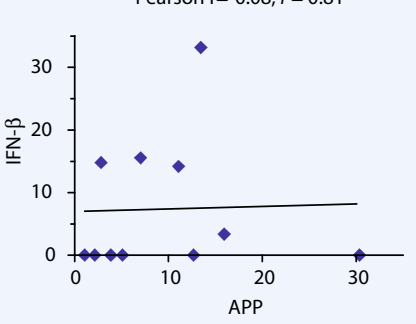

Pearson $\mathrm{r}=0.033 ; P=0.92$
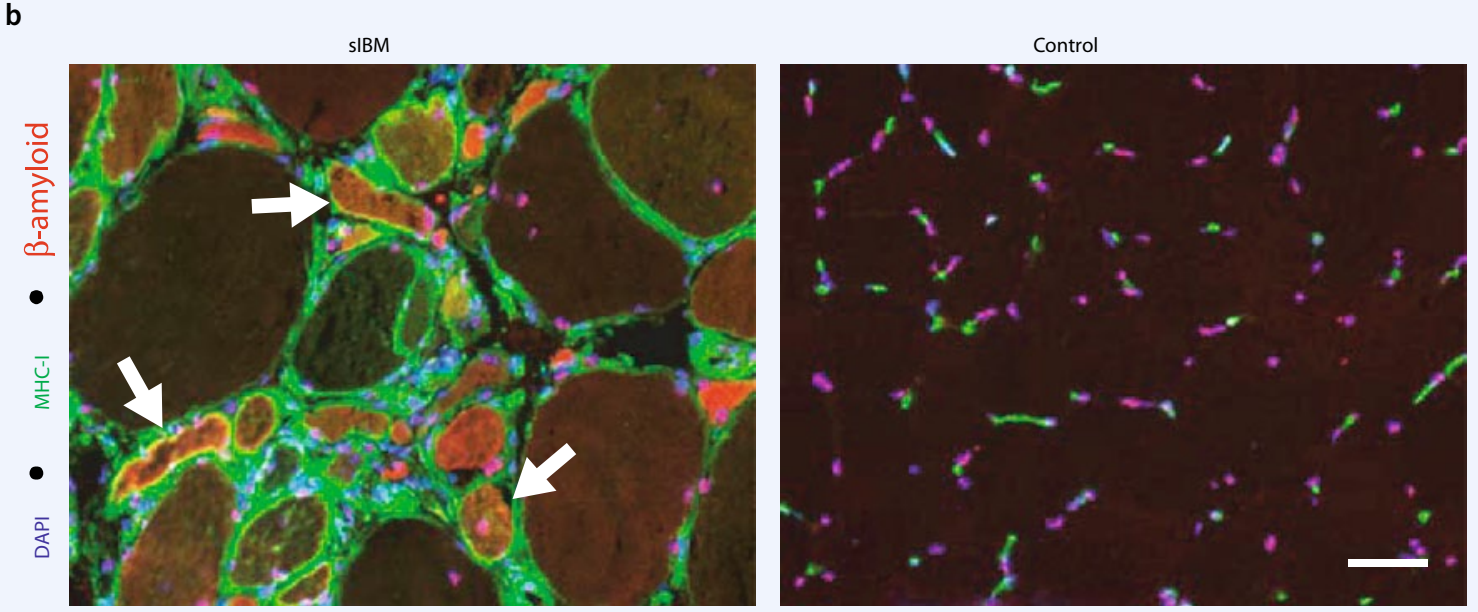

Fig. $3 \Delta$ a Correlation of mRNA expression of APP with relevant inflammatory markers such as the degree of inflammation in H\&E sections, as well as mRNA expression of chemokines and cytokines (mRNA data from $\bullet$ Fig. 2). b Immunohistochemical double staining for MHC-I (cell membrane) and $\beta$-amyloid (sarcoplasm) in a representative muscle of an sIBM patient (DAPI nuclear counterstaining). Arrows indicate muscle fibres which are double-positive for MHC-I and $\beta$-amyloid. (Images obtained from the open-access publication [23] with permission)

clear co-staining for $\beta$-amyloid ( $81 \%$ of fibres) be seen; none, however, was seen in controls. Taken together, these data indicate that autophagic breakdown of APP/ $\beta$-amyloid could play a role in the development of sIBM. However, it remains unclear whether this mechanism has a protective or rather a destructive function in SIBM, since autophagy can also occur alongside programmed cell death (apoptosis) [26].

\section{The association between inflammation and $\beta$-amyloid in muscle}

In addition to the above-mentioned degeneration with increased development of $\beta$-amyloid and inflammatory cell migration, a multitude of inflammatory mediators are formed in sIBM, which are each able to direct a specific cellular immune response to the muscle (overview in [6]), in particular the $\mathrm{CC}$ and $\mathrm{CXC}$ chemokines CCL-3, CCL-4, CXCL-9 and CXCL-10. In addition, both immune cells and muscle fibres themselves form inflammatory me- 
a

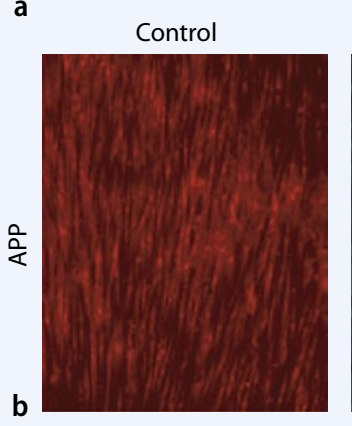

IL-1 $\beta$

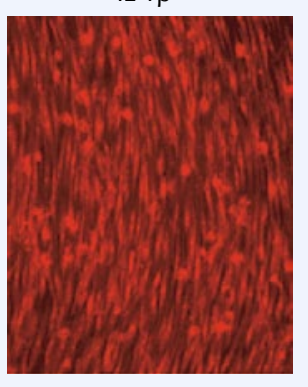

$\mathrm{IFN}-\gamma+\mathrm{IL}-1 \beta$

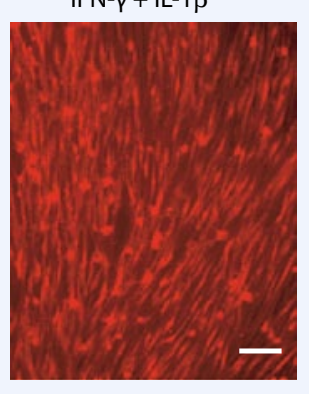

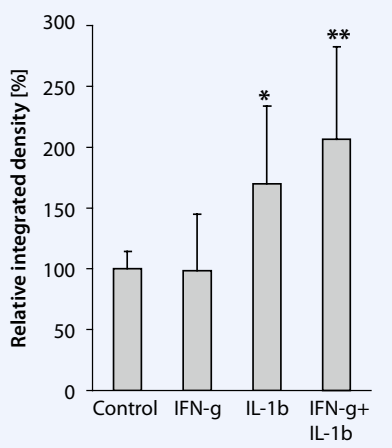

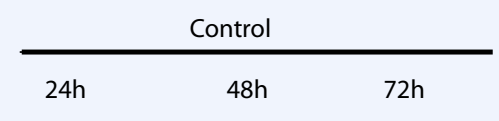

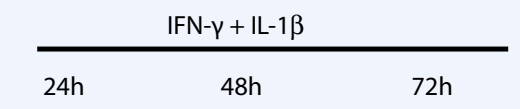

C

APP

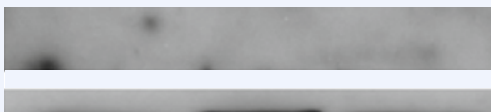

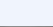

(8)

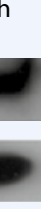

Fig. 4 Human primary muscle cultures following exposure to IFN- $\gamma$ and IL$1 \beta$. Striking upregulation of protein expression of APP after 24-72 $\mathrm{h}$ can be seen using immunocytochemistry (a) and Western Blot (b). $\beta$-amyloid accumulation is shown by thioflavin-S staining after $48 \mathrm{~h}$ (c). (Images obtained from the open-access publication [23] with permission)

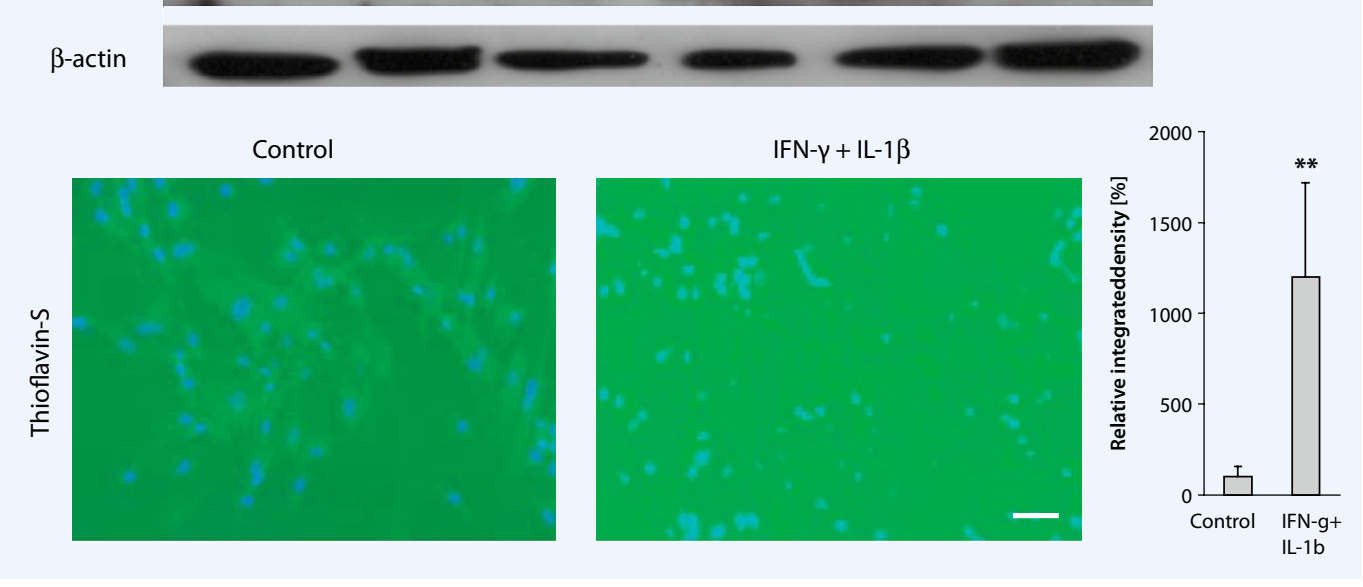

diators (cytokines), such as IL-1 $\beta$, TNF- $\alpha$ and TGF- $\beta$ (overview in [25]). In a recently published work, our group investigated the mRNA level of the relevant disease markers in sIBM and compared this with dermatomyositis and polymyositis, the other inflammatory muscle diseases. Using quantitative (real-time) PCR, it could be seen that significantly higher levels of chemokines CXCL-9, CCL-3 and CCL-4, as well as cytokines IFN- $\gamma$, TNF- $\alpha$ and IL$1 \beta$ were expressed in sIBM compared with healthy control muscle (• Fig. 2a).

Moreover, the mRNA level in sIBM was in most cases markedly higher than in polymyositis or dermatomyositis. Tissue staining showed that the protein levels of inflammatory markers such as MHCI, CXCL-9, CCL-3 and IL- $1 \beta$ in sIBM were also each formed by the muscle fibres themselves, whereas in polymyositis/ dermatomyositis these molecules are primarily formed by inflammatory cells or in connective tissue, or by blood vessels
(• Fig. 2b). Markers which were associated with amyloid, cell stress or degeneration/regeneration, such as APP, $\alpha \mathrm{B}$-crystallin, desmin or NCAM were also increased in sIBM compared with controls, however not compared with polymyositis or dermatomyositis. Further investigation of mRNA data showed that APP as the major degeneration molecule correlated significantly with the relevant inflammatory markers CXCL-9, CCL-3, CCL-4 and IFN- $\gamma$ (• Fig. 3a). No similar correlation could be found for polymyositis and dermatomyositis. Tissue staining provided precisely the same evidence of an association between inflammation and degeneration: The inflammatory marker MHC- 1 was found in the same muscle fibres which were also positive for $\beta$-amyloid (• Fig. 3b). On a functional level, it could be demonstrated that the cytokine IL- $1 \beta$ in primary human muscle cultures caused upregulation of APP (• Fig. 4a). Additional stimulation with
IFN- $\gamma$ increased this effect, which is likely explained by increased formation of the binding molecule (receptor) for IL-1 $\beta$ on the surface of the muscle cell. This up-regulation could be confirmed by Western Blot and the time course of this increase more accurately investigated (• Fig. 4b). There was also an increase in the ultimately depositing molecule, amyloid, in these muscle cells (• Fig. 4c).

The molecular mechanism of the association between $\beta$-amyloid and IL-1 $\beta$ in muscle has not been investigated in depth as yet. BACE1 has been considered in this context, the production or activation of which can also be increased under inflammatory conditions and lead to increased breakdown of $\beta$-amyloid from APP, as could already be shown in other cell systems [11]. A further mechanism which could explain the association between inflammation and degeneration in SIBM is autophagy. As mentioned above, new data indicate that vacuoles play a role in the 


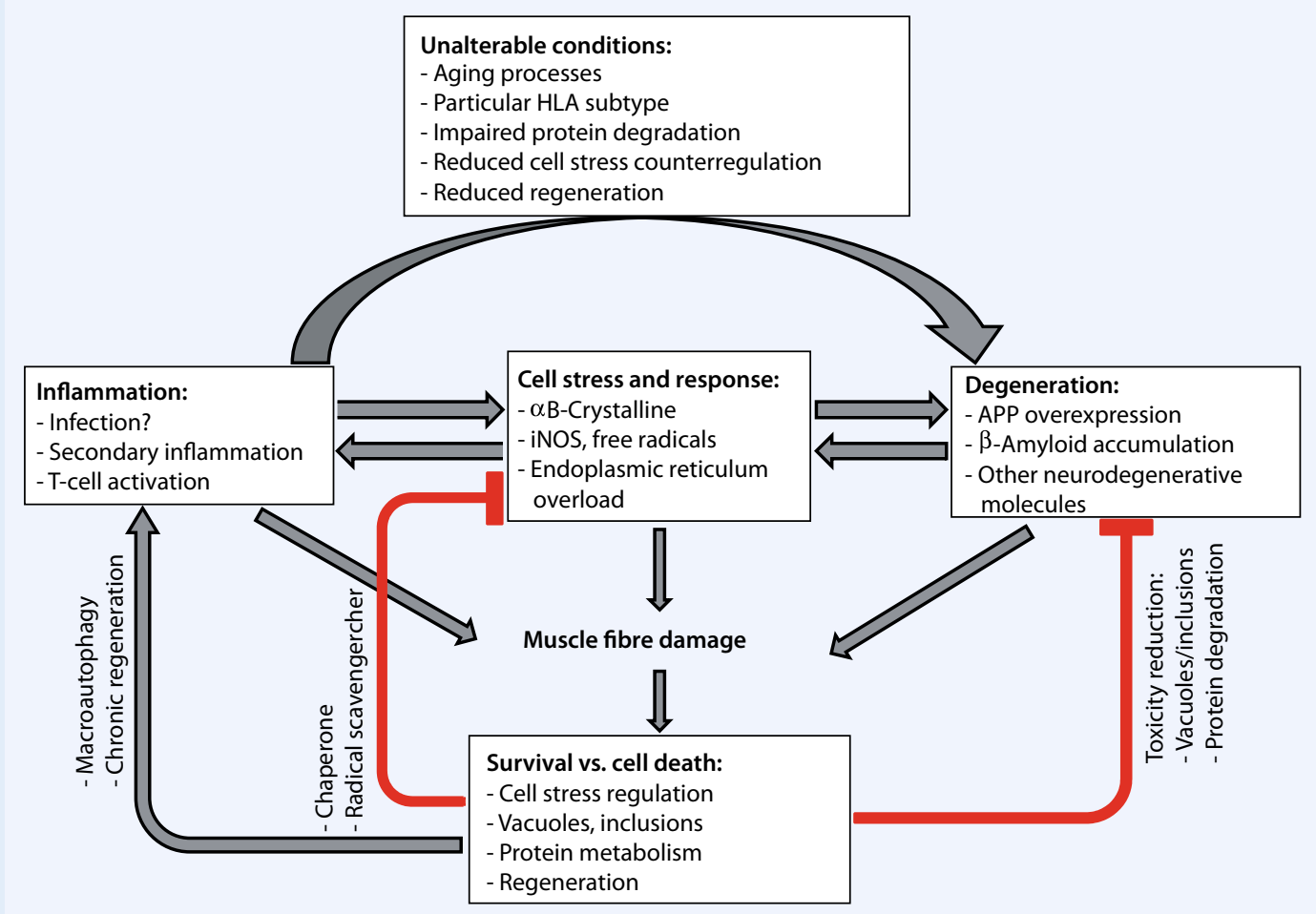

Fig. $5 \Delta$ Current model of the possible mechanisms of sIBM. Upon infection or other mechanism of damage to the muscle, unalterable conditions such as genetic factors may be the reason for an inflammatory reaction to cause degeneration with increased APP production and $\beta$-amyloid deposition. At the same time, there is a cell stress reaction that can, in turn, cause increased degeneration on the one hand while triggering or promoting an inflammatory reaction on the other. Following muscle damage, it is dependent upon certain opposing mechanisms as to whether the fibre survives or dies. Protective molecules such as chaperones can weaken a cell stress reaction or its effects on the cell, while compartmentalization of aberrant molecules such as $\beta$-amyloid in vacuoles or inclusion bodies may reduce its damaging effect. Increased protein metabolism may counteract progressive degeneration on the one hand; however, on the other increased inflammation is possible due to the presentation of antigens via the autophagy/MHC-Il pathway

generation of in $\beta$-amyloid in sIBM muscle. At the same time, it has been known for several years that the breakdown of proteins by means of macroautophagy can lead to their presentation as antigens by fragments reaching the cell surface via the MHC-II pathway [19]. In this way, cytokine-associated up-regulation of MHCII, as seen in muscle inflammation in sIBM patients, could simultaneously also cause the presentation of degeneration-associated molecules such as $\beta$-amyloid. To support this, a recent report demonstrated that, at $94 \%$, almost all muscle fibres which were double-positive for MHC-II and $\beta$-amyloid also contain a signal for $\mathrm{LC}_{3}$, thereby corresponding to macroautophagic vacuoles [14].

\section{Cell stress mechanisms}

One possible pathway for the association between inflammation and degeneration in sIBM muscle is cell stress. There are different types of cell stress, which can be caused by inflammation or the formation of free radicals. aB-crystallin has already been described as a cell stress molecule in muscle cells [8]: as a heat shock protein, $\alpha B$-crystallin can for example protect certain filament proteins such as actin or des$\mathrm{min}$ in a 'chaperone' function. Moreover, $\alpha \mathrm{B}$-crystallin has also been attributed a role in the elimination of $\beta$-amyloid and/ or its cell-damaging effects [8]. It could be shown in IBM muscle that aB-crystallin is present in fibres which demonstrate no abnormalities in terms of form or internal structure, i.e., which appeared morphologically normal [3]. These muscle fibres have been referred to as " $\mathrm{X}$ fibres", since it was assumed that they carry a very early pathological change, which would become manifest over time in the form of changes in cell structure, such as vacuoles for example. In a recently pub- lished work, our group was able to shown that $\alpha \mathrm{B}$-crystalline und $\beta$-amyloid correlate significantly with one another [16]. In particular, so-called "X fibres" were often positive for APP or even $\beta$-amyloid, which could also be shown together with other markers for regeneration or degeneration, such as NCAM or desmin in the same fibre. Fibres with vacuoles or other morphological signs of degeneration were also double-positive for $\alpha \mathrm{B}$-crystallin and $\mathrm{APP} / \beta$-amyloid. In functional investigations with muscle cultures it was found that the inflammatory cytokine IL-1 $\beta$ together with IFN- $\gamma$ caused up-regulation of $\alpha \mathrm{B}$-crystallin and simultaneously increased the expression of APP. Thus these data suggest an early cell reaction which can precede an accumulation of $\beta$-amyloid in sIBM muscle.

A second form of cell stress is the production of free radicals such as nitric ox- 
ide (NO), which can be produced by an inducible synthesis, iNOS.

A further association between a cell stress reaction and the accumulation of abnormal proteins in muscle comprises an endoplasmic reticulum cell stress reaction, e.g. in that the intracellular machinery forms a multitude of MHC-1 molecules. The negative effects of this endoplasmic reticulum cell stress reaction on muscle fibres has been observed in both animal models and cell culture systems [4][17]. Further investigations suggest that autophagic metabolization of proteins plays a particular role in this process [18][27].

To summarise, there are indications of an early cell stress reaction in sIBM muscle, which could be of significant relevance in the development of chronic inflammation as well as in the accumulation of abnormal proteins.

\section{Conclusion}

sIBM is the most frequently acquired muscle disease in patients over 50 years, causing progressive muscle loss with increasing weakness, which leads to reduced ability to walk. There is no effective treatment available to date, which is partly due to the fact that the cause is as yet unknown. - Fig. 5 shows a model of possible disease mechanisms, which include a specific inflammatory reaction and an accumulation of aberrant molecules in muscle fibres. The inflammatory mechanisms cause an increase in selfdestructive T-lymphocytes, which attack the muscle fibres. B-lymphocytes and a generation of self-reactive antibodies also appear to be involved. In the immune reaction in muscle, the fibres are able to play a role in determining the entire repertoire of the autoimmune cascade: (1) the production of chemokines contributes to the migration of immune cells into the muscle; (2) co-stimulatory molecules on the surface of muscle fibres could be involved in the presentation of auto-antigens; (3) the distribution of cytokines contributes to a long-lasting inflammatory milieu in that an increase in immune cells in the form of clonal expansion can take place. In addition to $\beta$-amyloid, there is increased deposition in the muscle of other molecules involved in degenerative diseases, such as tau and presenilin, among others. Autophagic mechanisms also play a role in the generation of $\beta$-amyloid and can simultaneously increase or trigger the inflammatory reaction. IL-1 $\beta$ is of particular importance in this inflammation, since it also causes increased production of APP und $\beta$-amyloid in muscle cells. Even before this inflammatory cascade and detectable degenerative mechanisms, there is production of cell stress molecules such as aB-crystallin, which indicates a possible dysfunction in molecules important for cell stress.

The data presented in this overview article demonstrate that our understanding of the cause of sIBM has improved considerably in recent years. However, significant associations remain incompletely understood. Only a better understanding of the disease mechanisms will make it possible to develop targeted and promising novel treatment strategies. Moreover, these mechanisms could contribute significantly to our understanding of neurodegenerative diseases.

\section{Acknowledgements}

Parts of the work presented in this overview were supported by the DFG (Schm 1669/1-1, 1669/2-1), the Association Française contre les Myopathies (AFM; AM/ NM/2006.1377/12087), Göttingen University (intramural research funding) and the "intramural program" of NINDS. I would like to thank all co-authors for the good collaboration, as well as Hanns Lochmüller for tissue samples of polymyositis patients which were made available over the muscle biobank (service structure $S_{1}$ of the BMBF-sponsored MD-NET).

\section{Corresponding address}

\section{PD Dr. J. Schmidt}

Department of Neurology

Robert-Koch-Str. 40, 37075 Göttingen

Germany

j.schmidt@med.uni-goettingen.de

J. Schmidt 1991-1997: Medical studies at Würzburg University; 1998-1999: Intern, Department of Neurology at Würzburg University; 2000: Doctorate with an experimental neuroimmunological work at the Department of Neurology, Würzburg University; 20002002: Research associate in the Neuroimmunology group, Würzburg University; 2002-2005: DFG Grant for post-doctoral research in the Neuromuscular Department, NINDS, NIH (USA); 2005-2008: Assistant doctor in the Department of Neurology, Göttingen Medical University (UMG); since 2005: Head of the research group Muscle Immunobiology, Department of Neurology, UMG; since 2008: Senior physician and head of the Clinical Neurophysiology laboratory of the Department of Neurology, UMG; since 2008: Head of the Neuromuscular Outpatient Section and spokesperson for the Neuromuscular Center, UMG; 2009: Postdoctoral lectureship at the UMG, venia legend for Neurology. Prizes and awards: 2003: Sobek Junior Prize (Dt. Multiple Sklerose Gesellschaft); 2005: Fellow recognition of exceptional mentoring (NINDS, NIH); 2009: Felix Jerusalem Prize (1st Prize; German Society for Muscle Disease).

\section{References}

1. Askanas V, Engel WK (2008) Inclusion-body myositis: muscle-fiber molecular pathology and possible pathogenic significance of its similarity to Alzheimer's and Parkinson's disease brains. Acta Neuropathol 116:583-595

2. Askanas V, McFerrin J, Baque S, Alvarez RB, Sarkozi E, Engel WK (1996) Transfer of beta-amyloid precursor protein gene using adenovirus vector causes mitochondrial abnormalities in cultured normal human Muscle. Proc Natl Acad Sci U S A 93:-1314-1319

3. Banwell BL, Engel AG (2000) Alpha b-crystallin immunolocalization yields new insights into inclusion body myositis. Neurology 54:1033-1041

4. Casciola-Rosen L, Nagaraju K, Plotz P, Wang K, Levine S, Gabrielson E, Corse A, Rosen A (2005) Enhanced autoantigen expression in regenerating muscle cells in idiopathic inflammatory myopathy. J Exp Med 201:591-601

5. Chou SM (1967) Myxovirus-like structures in a case of human chronic polymyositis. Science 158:14531455

6. De Paepe B, Creus KK, De Bleecker JL (2008) Chemokines in idiopathic inflammatory myopathies. Front Biosci 13:2548-2577

7. Dimitri D, Benveniste O, Dubourg O, Maisonobe T, Eymard B, Amoura Z, Jean L, Tiev K, Piette JC, Klatzmann D, Herson S, Boyer O (2006) Shared blood and muscle CD8+ T-cell expansions in inclusion body myositis. Brain 129:986-995

8. Goldfarb LG, Vicart P, Goebel HH, Dalakas MC (2004) Desmin myopathy. Brain 127:723-734

9. Griggs RC (2006) The current status of treatment for inclusion-body myositis. Neurology 66:S30-S32 
10. Hara T, Nakamura K, Matsui M, Yamamoto A, Nakahara Y, Suzuki-Migishima R, Yokoyama M, Mishima K, Saito I, Okano H, Mizushima N (2006) Suppression of basal autophagy in neural cells causes neurodegenerative disease in mice. Nature 441:885889

11. Hong HS, Hwang EM, Sim HJ, Cho HJ, Boo JH, Oh SS, Kim SU, Mook-Jung I (2003) Interferon gamma stimulates beta-secretase expression and SAPPbeta production in astrocytes. Biochem Biophys Res Commun 307:922-927

12. Kitazawa M, Green KN, Caccamo A, LaFerla FM (2006) Genetically augmenting abeta42 levels in skeletal muscle exacerbates inclusion body myositis-like pathology and motor deficits in transgenic mice. Am J Pathol 168:1986-1997

13. Komatsu M, Waguri S, Chiba T, Murata S, Iwata J, Tanida I, Ueno T, Koike M, Uchiyama Y, Kominami E, Tanaka K (2006) Loss of autophagy in the central nervous system causes neurodegeneration in mice. Nature 441:880-884

14. Lunemann JD, Schmidt J, Schmid D, Barthel K, Wrede A, Dalakas MC, Munz C (2007) Beta-amyloid is a substrate of autophagy in sporadic inclusion body myositis. Ann Neurol 61:476-483

15. Mizushima N (2007) Autophagy: process and function. Genes Dev 21:2861-2873

16. Muth IE, Barthel K, Bahr M, Dalakas MC, Schmidt J (2009) Proinflammatory cell stress in sporadic inclusion body myositis muscle: overexpression of alphaB-crystallin is associated with amyloid precursor protein and accumulation of beta-amyloid. J Neurol Neurosurg Psychiatry 80:1344-1349

17. Nagaraju K, Raben $N$, Loeffler L, Parker T, Rochon PJ, Lee E, Danning C, Wada R, Thompson C, Bahtiyar G, Craft J, Hooft VH, Plotz P (2000) Conditional up-regulation of MHC class I in skeletal muscle leads to self-sustaining autoimmune myositis and myositis-specific autoantibodies. Proc Natl Acad Sci U S A 97:9209-9214

18. Nagaraju K, Casciola-Rosen L, Lundberg I, Rawat R, Cutting S, Thapliyal R, Chang J, Dwivedi S, Mitsak M, Chen YW, Plotz P, Rosen A, Hoffman E, Raben $\mathrm{N}$ (2005) Activation of the endoplasmic reticulum stress response in autoimmune myositis - potential role in muscle fiber damage and dysfunction. Arthritis Rheum 52:1824-1835

19. Paludan C, Schmid D, Landthaler M, Vockerodt M, Kube D, TuschIT, Munz C (2005) Endogenous MHC class II processing of a viral nuclear antigen after autophagy. Science 307:593-596

20. Ravikumar B, Vacher C, Berger Z, Davies JE, Luo S, Oroz LG, Scaravilli F, Easton DF, Duden R, O'Kane CJ, Rubinsztein DC (2004) Inhibition of MTOR induces autophagy and reduces toxicity of polyglutamine expansions in fly and mouse models of Huntington disease. Nat Genet 36:585-595

21. Salajegheh M, Rakocevic G, Raju R, Shatunov A, Goldfarb LG, Dalakas MC (2007) T cell receptor profiling in muscle and blood lymphocytes in sporadic inclusion body myositis. Neurology 69:16721679

22. Schmidt J, Rakocevic G, Raju R, Dalakas MC (2004) Upregulated inducible co-stimulator (ICOS) and ICOS-ligand in inclusion body myositis muscle: significance for CD8(+) T cell cytotoxicity. Brain 127:1182-1190

23. Schmidt J, Barthel K, Wrede A, Salajegheh M, Bahr M, Dalakas MC (2008) Interrelation of inflammation and APP in SIBM: IL-1 beta induces accumulation of beta-amyloid in skeletal muscle. Brain 131:1228-1240
24. Sugarman MC, Yamasaki TR, Oddo S, Echegoyen JC, Murphy MP, Golde TE, Jannatipour M, Leissring MA, LaFerla FM (2002) Inclusion body myositis-like phenotype induced by transgenic overexpression of beta APP in skeletal muscle. Proc Natl Acad Sci U S A 99:6334-6339

25. Tournadre A, Miossec $P$ (2007) Cytokine response in inflammatory myopathies. Curr Rheumatol Rep 9:286-290

26. Tsujimoto Y, Shimizu S (2005) Another way to die: autophagic programmed cell death. Cell Death Differ 12[Suppl 2]:1528-1534

27. Vattemi G, Engel WK, McFerrin J, Askanas V (2004) Endoplasmic reticulum stress and unfolded protein response in inclusion body myositis muscle. Am J Pathol 164:1-7

28. Wiendl H, Mitsdoerffer M, Schneider D, Melms A, Lochmuller $\mathrm{H}$, Hohlfeld R, Weller M (2003) Muscle fibres and cultured muscle cells express the B7.1/2-related inducible co-stimulatory molecule, ICOSL: implications for the pathogenesis of inflammatory myopathies. Brain 126:1026-1035

29. Wiendl H, Hohlfeld R, Kieseier BC (2005) Immunobiology of muscle: advances in understanding an immunological microenvironment. Trends Immunol 26:373-380

30. Yunis EJ, Samaha FJ (1971) Inclusion body myositis. Lab Invest 25:240-248 